\title{
The Norwegian Motion-Laboratory
}

\author{
Sondre Sanden Tørdal, ${ }^{1}$ Jan Thomas Olsen ${ }^{1}$ and Geir Hovland ${ }^{1}$
}

${ }^{1}$ Faculty of Engineering and Science, University of Agder, N-4898 Grimstad, Norway. E-mail: sondre.tordal@uia.no

\begin{abstract}
This paper contains an overview of the equipment currently available in the Norwegian Motion Laboratory, a description of the IT networking infrastructure in the laboratory, a GitHub link to open source code developed, description of the PyQt-based graphical user interface, presentation of robot forward and inverse kinematics, presentation of equations of motion for the suspended load motion and a description of the full system kinematics. The paper ends with a list of research experiments and publications from the laboratory to date.
\end{abstract}

Keywords: Motion Compensation, Qualisys, Leica, Bosch-Rexroth, Comau, Robotics, Mechatronics, Lab Setup, Experiments, and Stewart Platforms.

\section{Introduction}

Research and development of new solutions related to the area of offshore motion systems heavily rely on experiments and test scenarios. Opposed to conducting experiments in complex and costly offshore environments, access to a scaled testing facility can prove to be beneficial when concerning safety, efficiency, implementation, and cost.

The development of the Norwegian Motion Laboratory started in 2010 with the first Stewart Platform and this paper summarizes the efforts since then to build up this laboratory to the facility it has become today. The motion laboratory was originally developed as part of NORCOWE (Norwegian Center for Offshore Wind Energy) and operated by the University of Agder (UiA), Grimstad, www.motion-lab.no. Today the laboratory is heavily used by the research center SFI Offshore Mechatronics, and the laboratory is on Norwegian Roadmap for Research Infrastructure, Norwegian Research Council (2016). The facility aims towards extending and improving the current methods and solutions related to offshore motion systems and allows for research and experiments to be conducted in a controlled and safe environment. The available equipment in the motion laboratory allows for vari- ous experiments to be conducted, this includes; motion compensation, accuracy performance evaluation of motion sensor equipment, wave-induced motion simulation, where the current ongoing research involves the field of motion compensation.

Advanced motion compensation is a topic of engineering with increased interest from the offshore industry. Problems concerning complex and autonomous load handling scenarios are expected to increase in the future. Operations involving installations of floating wind turbines, autonomous shipping, and vessel-tovessel loading are a few examples of applications where motion compensation is beneficial for increased performance and safety.

Research and detailed studies of the system's kinematics is essential for the development of a motion compensation system. Safe maneuvering of the cargo from one floating vessel to another requires a full analysis of the kinematic links related to the suspended load, load handling equipment, and the wave-induced relative vessel motions. The resulting kinematic solution based on geometric transformation can be combined with the measuring and tracking system allows for the development of a suitable control system capable of transporting the cargo in a safe and efficient manner.

This paper concerns the configuration setup and 
functionality of the Norwegian Motion Laboratory (Motion-Lab). A brief overview of the available equipment and their related specification will be presented together with the communication configuration and control interface. A kinematic analysis of the different equipment found in the lab will be presented according to the test facility setup.

\section{Experimental Lab Setup}

The Norwegian Motion-Laboratory (Norwegian Motion Laboratory, 2018) is a research facility located at the University of Agder (UiA) campus Grimstad. The establishment was possible due to funding associated with the Norwegian Center for Offshore Wind Energy (NORCOWE), together with additional funding from UiA and the Research Council of Norway. The available setup and equipment enable the laboratory to function as a unique testing facility to develop and improve methods of offshore motion compensation and other motion-related tasks in general. The main equipment of the Motion-Lab are the two Stewart Platforms (SPs), these are installed to simulate two independent vessels exposed to stochastic wave motion. An industrial robot, equipped with an electrical winch, is installed on top of the largest platform. This configuration enables the lab to be used in experiments concerning vessel-to-vessel motion compensation (VVMC), where the larger SP will act as the main vessel, and the smaller SP represents the vessel for which the cargo can be transported to. The establishment is equipped with several sensors and tracking equipment allowing for a full measurement coverage of the involved motion systems. Figure 1 shows a photo of the main equipment in the Motion-Lab.

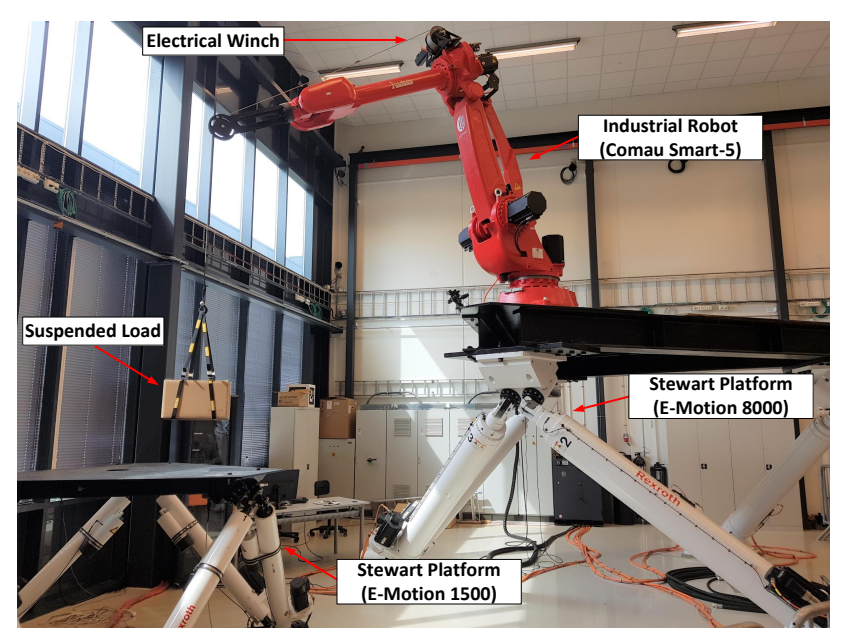

Figure 1: An overview of the experimental setup at the Norwegian Motion-Laboratory.

\subsection{Stewart Platforms}

The two installed SPs are both manufactured by Bosch Rexroth, hence the specific models are the E-Motion 8000 and the E-Motion 1500. These platforms are actuated by six prismatic electrical actuators capable of simulating 6 Degrees of Freedom (DOF) motions. The largest of the two platforms E-Motion 8000 (EM 8000) has a payload capacity of $5500[\mathrm{~kg}]$, and the smaller platform E-Motion 1500 (EM 1500) has a payload capacity of $1500[\mathrm{~kg}]$. Technical specifications and capacity of the SPs can be found in Appendix A, Table 8.

\subsection{Industrial Robot}

A high-performance industrial robot (Comau SMART5 NJ 110-3.0) is used to simulate the load handling equipment capable of performing the motion compensation tasks. The Comau robot is a 6-DOF anthropomorphic manipulator, with a wrist payload capacity of $110[\mathrm{~kg}]$, and a maximum horizontal reach at appropriately $2.5[\mathrm{~m}]$. To closer resemble the configuration of an offshore loader crane, it is possible to lock the wrist joints of the robot, hence removing 3-DOF. Technical specification related to the Comau robot is available in Appendix A Table 6.

\subsection{Electrical Winch}

An electrically actuated winch is installed on top of the Comau robot, this configuration enables simulations of the lifting operations related to load handling scenarios. A servomotor from Beckhoff (AM8532-H) (Beckhoff, 2018b) is used to actuate the winch drum, and a digital servo drive from Beckhoff (AX5103) (Beckhoff, 2018a) is installed to control the servomotor using the EtherCAT Protocol. This configuration allows the winch system to be fully controllable via Beckhoff's PLC programming interface using the Structured Text language. Technical information related to the servomotor can be found in Appendix A, Table 9.

\subsection{Motion Reference Unit}

Experiments related to VVMC scenarios have heavily relied on real-time detection of the relative motion of the vessels. Each of the SPs is equipped with a motion reference unit (MRU) from Kongsberg Seatex (Kongsberg Seatex, 2018) (MRU H 5th generation). These sensors incorporate both a three-axis accelerometer and a rate gyroscope, which enable for real-time measurements of the two Stewart platforms position, velocity, and acceleration. Both of the MRU's are connected to a central control unit, enabling for easy access 
of the detected motion data. Figure 2 shows a photo the MRU installed on the E-Motion 1500 platform.

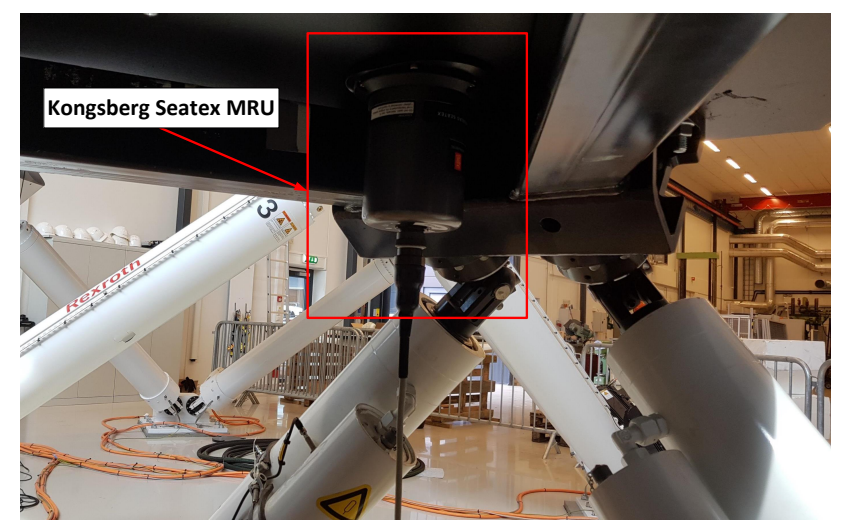

Figure 2: Kongsberg Seatex MRU installed on E-Motion 1500 platform.

\subsection{Motion-Capture System}

A real-time motion-capture system consisting of 17 Oqus 700+ cameras from Qualisys (Qualisys, 2018) are installed in the Motion-Lab. The cameras are mounted on the surrounding walls, providing full coverage of the laboratory's working volume. Using retro-reflective markers, the cameras can track objects of various kind depending on the application and experimental setup. Table 1 describes the two available capture-modes of the cameras. Figure 3 shows photos of the available motion-capture system.

Table 1: Qualisys Qqus 700+ capture-modes

\begin{tabular}{lcc}
\hline & Normal & High-speed \\
\hline Frames per second & 300 & 1100 \\
Megapixels & 12 & 3 \\
Resolution & $4096 \times 3072$ & $2024 \times 1536$ \\
\hline
\end{tabular}
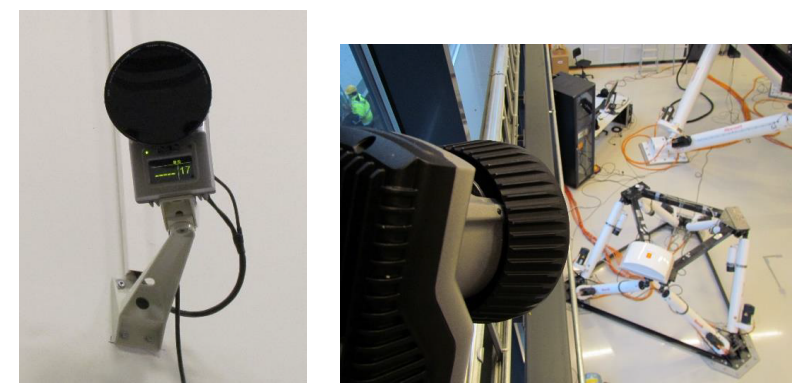

Figure 3: Qualisys motion-capture system, Oqus 700+ cameras installed in the Motion-Lab.

\subsection{Laser Tracker}

A Leica Absolute Tracker (AT960) and a Leica T-Mac Frame (TMC30-F) are available in the Motion-Lab. The laser tracker can provide real-time 6-DOF highprecision probing and measurements, with a maximum permissible error (MPE) of $\pm 15 \mu m+6 \mu m / m$ and a measuring range up to $160[\mathrm{~m}](\varnothing)$ (Hexagon Manufacturing Intelligence, 2018). Combining the laser tracker with the available Leica T-Mac measuring probe enables real-time tracking capabilities in 6-DOF, where the tracker can dynamically follow the probing frame. Utilizing the EtherCAT protocol, the combined system allows for delivery of high-speed deterministic measurements at a sampling rate up to $1[k H z]$. The laser tracker and probing frame are depicted in Figure $4 \mathrm{a}$ and $4 \mathrm{~b}$, respectively.

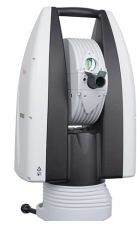

(a) Leica Absolute Tracker (AT960)

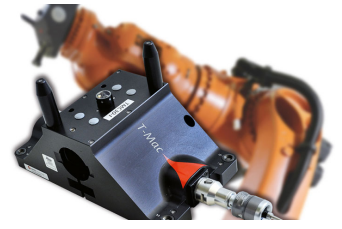

(b) Leica T-Mac Frame (TMC30-F)
Figure 4: Leica 6-DOF measurement system (Hexagon Manufacturing Intelligence, 2018).

\subsection{Central Control and Processing Unit}

An industrial PC, namely the Beckhoff CX2040 (Beckhoff, 2018a) is assigned as the central control and processing unit for the installed equipment in the Norwegian Motion Laboratory. The employed control unit is a combination of an embedded PC and a hardware PLC, running a Windows 7 Embedded operating system (OS). The system is compatible with Beckhoff's TwinCAT 3 automation software (Beckhoff, 2018), which enables the unit to be configured with a real-time runtime kernel running in parallel with the Windows OS. This feature, which is known as TwinCAT - eXtended Automation Runtime (XAR) (Beckhoff, 2018) eliminates the possibility of normal OS interrupts, hence guaranteeing a real-time control and monitoring system for the connected equipment. The Beckhoff control unit utilized for the experiments conducted in the Motion-Lab is depicted in Figure 5 


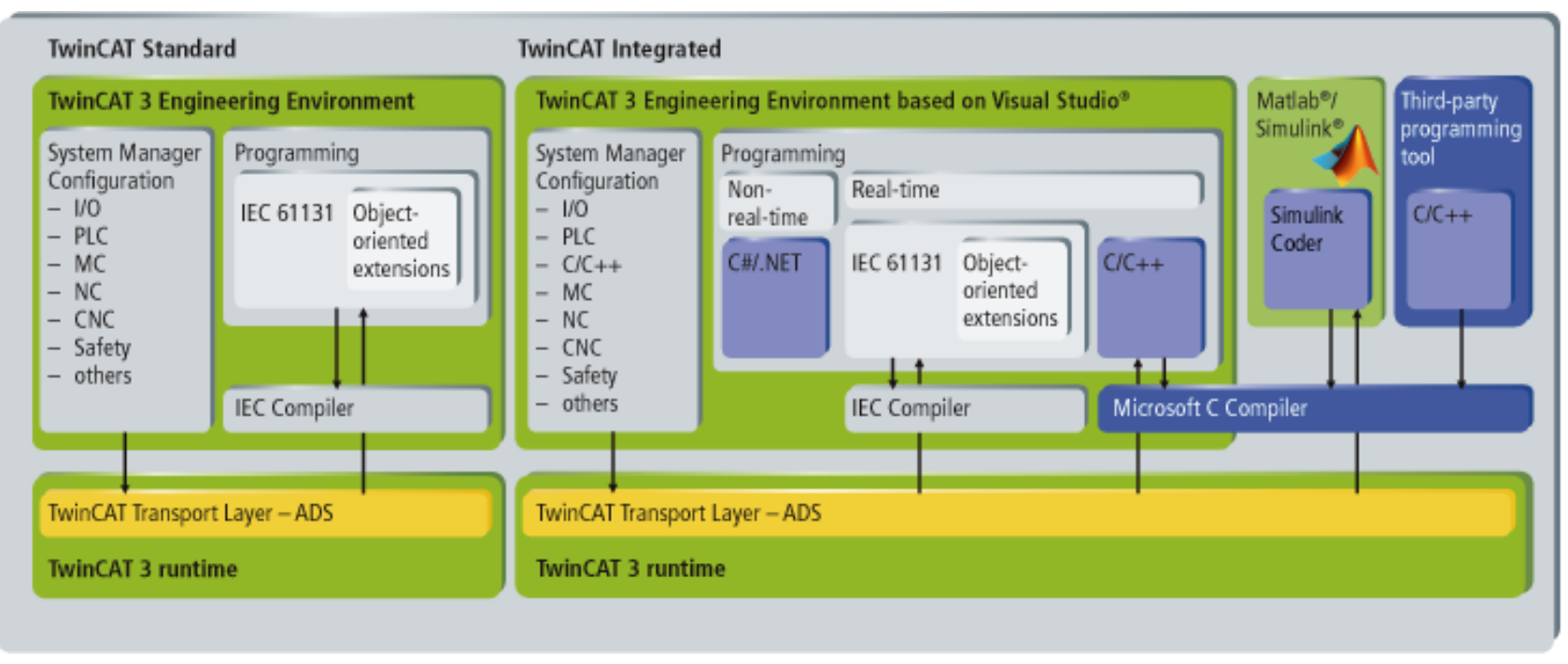

Figure 6: Overview of the TwinCAT 3 XAE capabilities and features (Beckhoff, 2018).

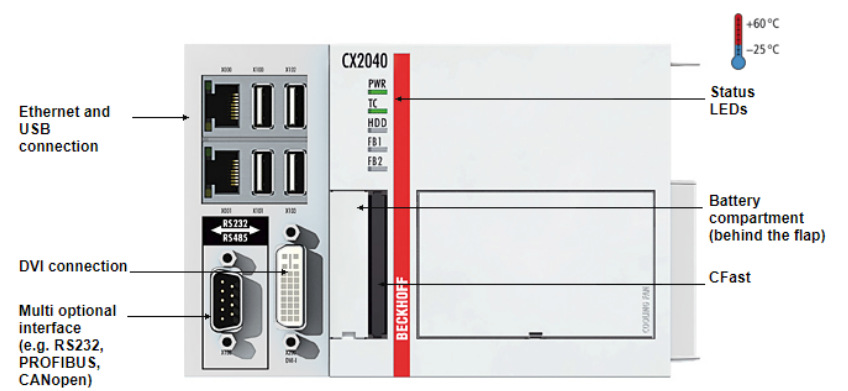

Figure 5: The central control unit, Beckhoff CX2040 (Beckhoff, 2018b).

The eXtended Automation Engineering (XAE) (Beckhoff, 2018) is also available via the TwinCAT software, this feature acts as an extension to Microsoft Visual Studio which allows the development and debugging to be conducted on an independent Windows computer using the programming languages defined by the IEC 6113-3 standard. Figure 6 shows an overview of the TwinCAT XAE capabilities.

The connected equipment communicates with the control unit either via an Ethernet or EtherCAT connection or directly through the I/O modules. The Beckhoff unit utilizes the ADS interface, which allows for data to be transmitted and received by the control unit while running a real-time runtime kernel. Hence, enabling the possibility for data-transfer between a real-time application running on the control unit and a non-real-time system such as a development computer (Windows or a Linux computer etc.), by sending and receiving data via the ADS communication protocol. Such a feature can typically be integrated with a userinterface control and a live-update of monitoring data.
An illustration of TwinCAT's ADS communication layout and development capabilities is shown in Figure 7.

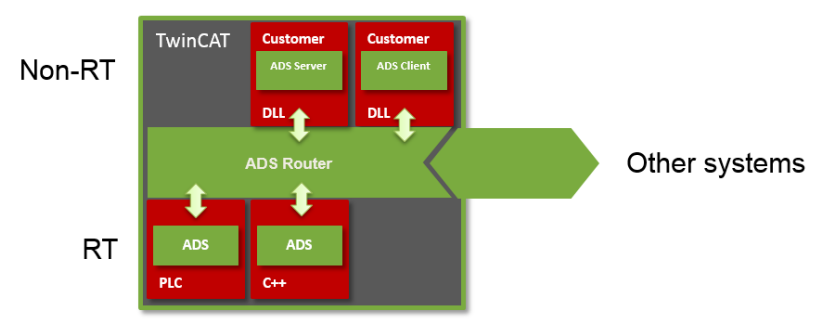

Figure 7: Illustration of the TwinCAT ADS communication bridge (Beckhoff, 2018).

As mentioned the Beckhoff CX2040 possesses functionality of a hardware PLC i.e. the control unit has available slots for installation of extension modules, where the CX2040 installed in the Motion-Lab is equipped with a total of six I/O modules; two analog output modules, two analog input modules, one digital output module, and one digital input module. In addition, several Ethernet interface (RJ45 sockets) modules are installed to enable communication with the connected equipment and sensors. Figure 8 illustrates the IO modules which are available from the central control unit.

\subsection{Connection Configuration}

The Motion-Lab consists of multiple different systems and equipment, this includes several dedicated computers responsible for the connection and interaction with the available devices. The current configuration of the Motion-Lab consist of a total of six computers: 


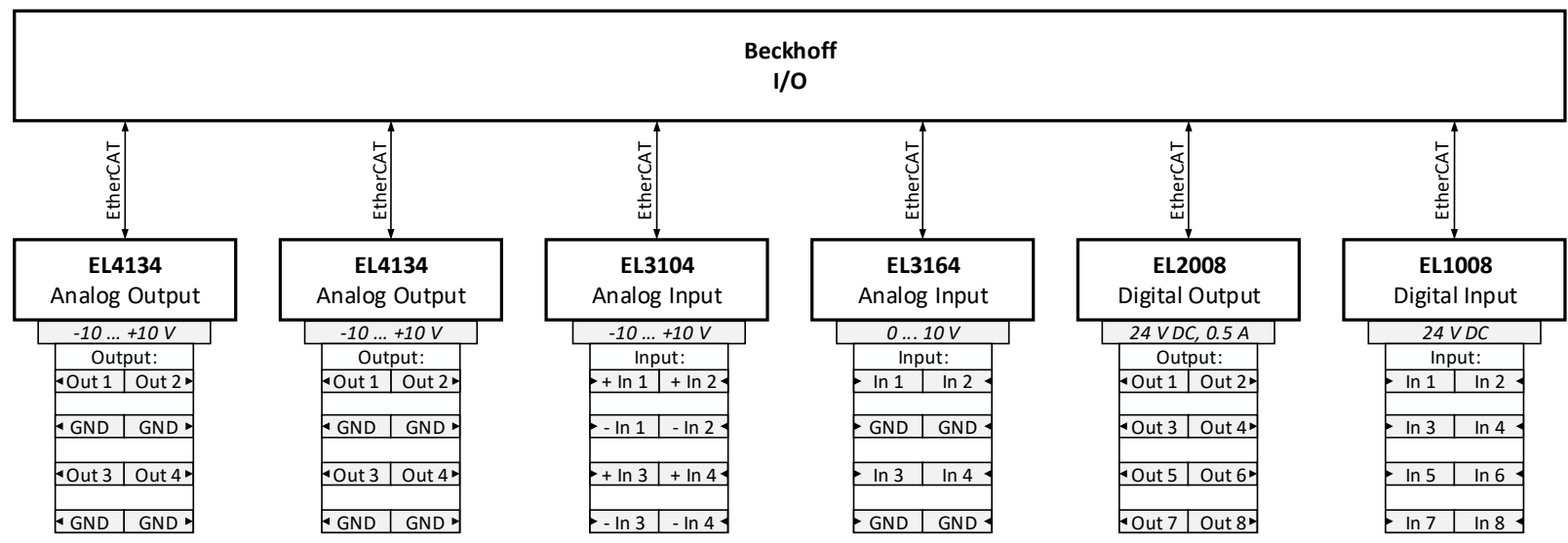

Figure 8: Overview of the available Analog and Digital I/O Modules of the Beckhoff CX 2040 control unit.

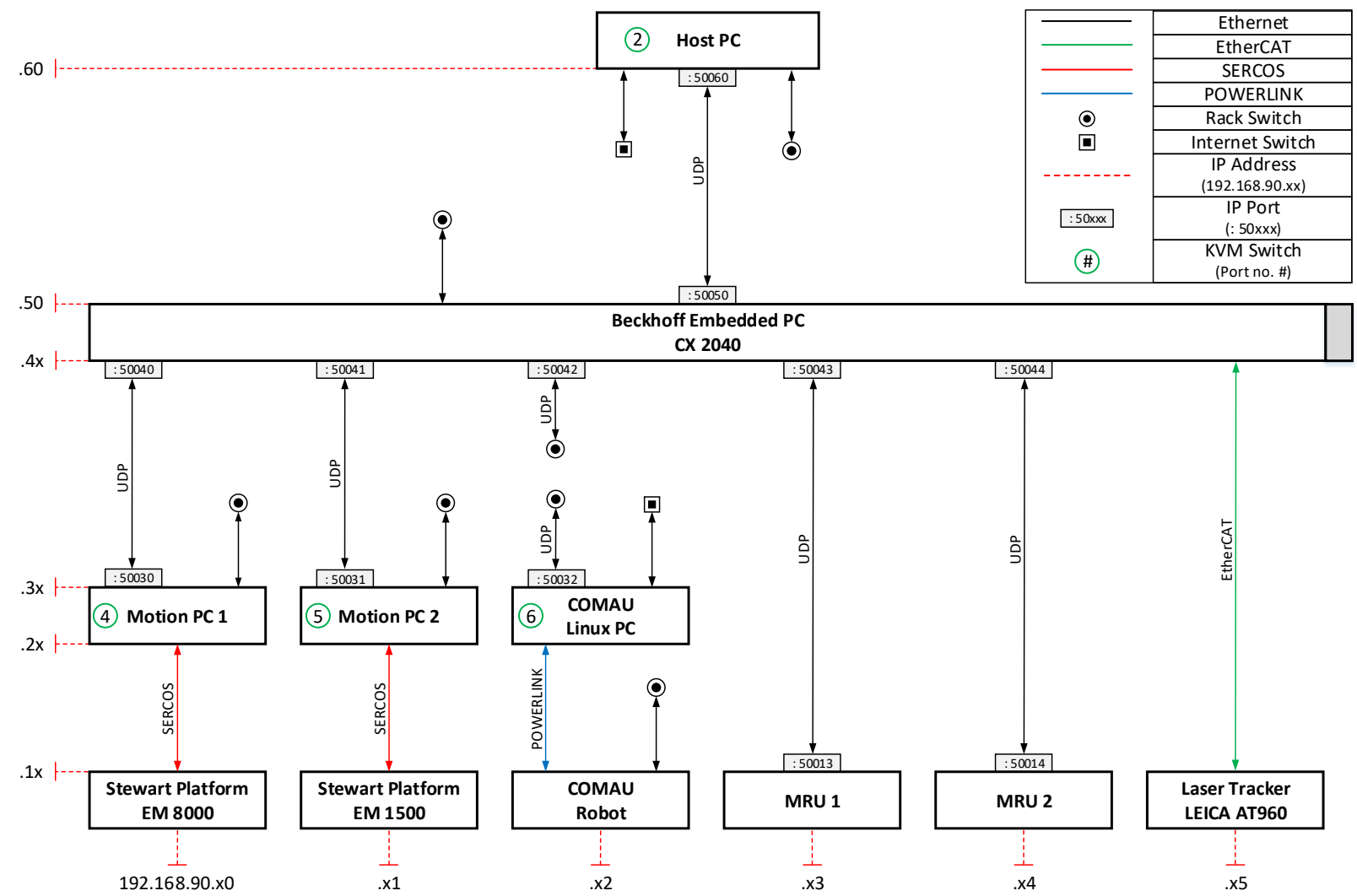

Figure 9: Overview of the connection configuration for the equipment in the Norwegian Motion-Laboratory. 
- The Beckhoff embedded PC/PLC (CX2040) which acts as the overall central control unit.

- Two motion PC's responsible for interaction with each of the SPs.

- A Linux based system used for managing the Comau robot.

- A dedicated motion-capture computer for the Qualisys Oqus motion-capture system.

- A development computer, running a standard Windows 10 OS with TwinCAT XAE (referred to as the Host PC).

A schematic overview of the Motion-Lab's connection configuration is shown in Figure 9. The lowest level represents the main equipment of the laboratory, where the connection to the central control unit is either direct or indirect via dedicated computers.

As the figure illustrates, different connection type uses different communication protocols, distinguished by the color of the vertical connection lines. E.g. the SPs use a SERCOS communication protocol to interact with the motion PCs.

All of the installed equipment in the Motion-Lab have been assigned at least one or more static IP address and communication port. All of the assigned IP addresses are on the form [192.168.90.xx] where the two last digits represent the row and column number, respectively, illustrated in the schematic as dashed red lines. E.g. the Comau robot, located at the first row and the third column has been assigned with [192.168.90.12] as the IP address (the column number starts at 0 ). Some of the installed equipment are assigned with multiple IP addresses, e.g. Motion PC 2 has been assigned with [192.168.90.21] for the interface with the EM1500, and [192.168.90.31] is allocated for the UDP communication with the central control unit (Beckhoff CX2040).

In addition to the direct connections, two network switches are installed in the communication configuration. The Internet Switch (distinguished by the square connection point in the schematic overview) offers a standard internet connection using dynamic IP addresses. The available Rack Switch is installed to easier enable internal interaction between the equipment and the development computer (Host PC), this connection is illustrated in the schematic overview by the circular connection point. Equipment connected to the Rack Switch is assigned with an additional static IP address on the form [192.168.90.1xx], where the two last digits represent the row and column number, respectively.
As an example, Motion PC 1 is configured with three different communication interfaces, [192.168.90.20] is assigned for interaction with the EM 8000 platform, [192.168.90.30] is used for UDP communication with the central control unit, and [192.168.90.130] is used for file transfer and SSH connection.

To easier access and interact with the computers in the Motion-Lab, a Keyboard-Video-Mouse (KVM) switch is installed in the rack containing all the PCs and the central control unit, this system allows the user to interact with each computer by a single master keyboard, monitor and mouse setup. Each computer is assigned with a KVM switch number, distinguished by the numbers outlined by green circles in the schematic communication overview. The switching between the different PCs using the KVM switch is carried out by pressing the Scroll Lock twice followed by the number representing the specific computer.

\subsection{Control Interface}

User interaction with the various equipment and functionality of the Norwegian Motion-Laboratory is available through a developed common control interface. A human machine interface (HMI) is designed using the Qt-designer and Python's PyQt5 library (Riverbank Computing Limited, 2018), where several predefined modes, interactive buttons, and graphical displays are implemented for efficient and safe use of the Motion-Lab equipment. The interface is available via the development computer, where the HMI interacts with the real-time runtime application on the central control unit, using the ADS communication for signal commands and data transmission. The main interface of the Motion-Lab HMI is depicted in Figure 10, which shows interactive modes to initiate motion of the SPs

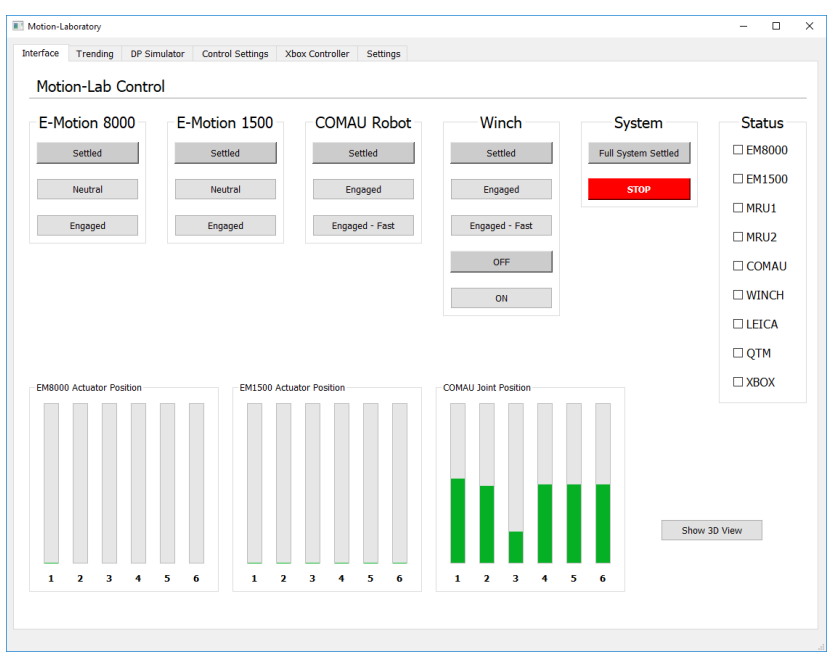

Figure 10: Main interface of the Motion-Lab HMI. 
and the Comau robot, as well as update-display of the position of the actuators.

The common control interface utilizes tabs for maneuvering between the implemented functionality. Figure 11 pictures one of the monitoring tabs of the HMI, where the user is presented with updating displays and graphical plots of the SP motion.

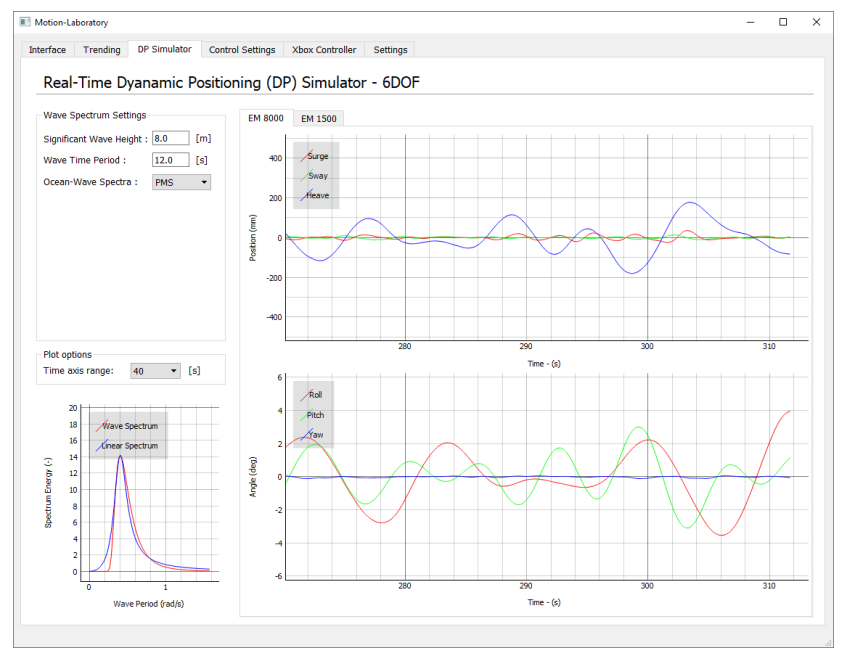

Figure 11: Monitoring tab of the HMI with updating displays and graphical plots.

\section{GitHub Library}

All code used to control and interfacing the Motion Laboratory is made available on GitHub on the following web address https://github.com/sondre1988/ motion-lab. To install the library, please clone the library by executing the following Git command:

1 git clone github.com/sondre1988/motion-lab and add both the folders matlab and simulink to the MATLAB search path. After adding these folders to the search path, the code snippets mentioned later on will be functional, and hence also all the code related to the HMI, and the PLC programs for the Beckhoff are available in their respective folders.

\section{Robot Kinematics}

\subsection{Geometry and Dimensions}

The industrial robot acts as the load handling equipment, the Comau robot utilizes a parallelogram linkage design, where the elbow actuator is positioned at the shoulder of the robot. The kinematics of this type of robots is well known but included in this paper for completeness. Figure 12 shows the geometric configuration of the robot with the extended arm attached to the tool point keeping the wire connected to the winch in place during operation. The closed-chain of the parallelogram can be simplified by considering the elbow actuator to be positioned at the elbow joint, hence allowing the robot to be treated as an open-chain serial manipulator. This simplification is considered to be valid due to the parallelogram linkage is kinematically equivalent to a two-link planar arm (Siciliano et al., 2010). Dimension values of the robot geometry is available in Appendix A, Table 7.

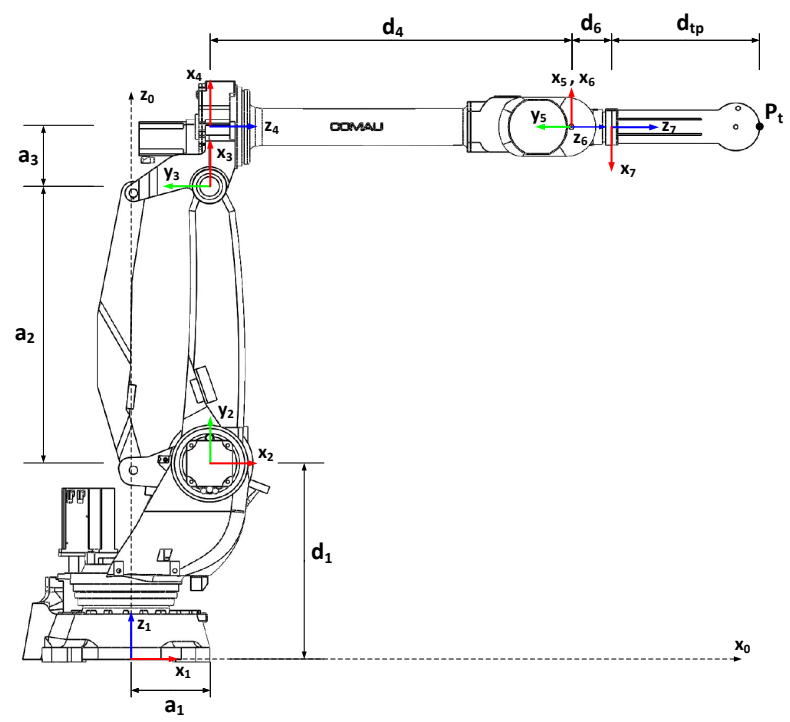

Figure 12: Geometric overview of the Comau Smart-5 NJ-110-3.0.

In experiments where the Comau robot is used to simulate a 3 -DOF offshore loader crane, the wrist joints $\theta_{4} \cdots \theta_{6}$ are fixed and equal to zero, hence removing the last $3-\mathrm{DOF}$ of the original 6 -DOF robot.

\subsection{Forward Kinematics}

The robot's forward kinematics is derived using the Denavit-Hartenberg (DH) convention (Denavit and Hartenberg, 1955; Hartenberg and Denavit, 1964). The rigid motion between joint $\theta_{i-1}$ and $\theta_{i}$ is expressed by the homogeneous transformation matrix $A_{i}$, which is constructed as a product of four basic transformations:

$$
A_{i}=R_{z}\left(\theta_{i}\right) T_{z}\left(d_{i}\right) T_{x}\left(a_{i}\right) R_{x}\left(\alpha_{i}\right)
$$


where:

$$
\begin{aligned}
& R_{z}\left(\theta_{i}\right)=\left[\begin{array}{cccc}
c_{\theta_{i}} & -s_{\theta_{i}} & 0 & 0 \\
s_{\theta_{i}} & c_{\theta_{i}} & 0 & 0 \\
0 & 0 & 1 & 0 \\
0 & 0 & 0 & 1
\end{array}\right] T_{z}\left(d_{i}\right)=\left[\begin{array}{cccc}
1 & 0 & 0 & 0 \\
0 & 1 & 0 & 0 \\
0 & 0 & 1 & d_{i} \\
0 & 0 & 0 & 1
\end{array}\right] \\
& R_{x}\left(\alpha_{i}\right)=\left[\begin{array}{cccc}
1 & 0 & 0 & 0 \\
0 & c_{\alpha_{i}} & -s_{\alpha_{i}} & 0 \\
0 & s_{\alpha_{i}} & c_{\alpha_{i}} & 0 \\
0 & 0 & 0 & 1
\end{array}\right] T_{x}\left(a_{i}\right)=\left[\begin{array}{cccc}
1 & 0 & 0 & a_{i} \\
0 & 1 & 0 & 0 \\
0 & 0 & 1 & 0 \\
0 & 0 & 0 & 1
\end{array}\right]
\end{aligned}
$$

where $\theta_{i}$ is the revolution angle of joint $i, d_{i}, a_{i}$ and $\alpha_{i}$ is referred to as the link length, joint offset and link twist respectively. $c_{k}$ and $s_{k}$ are abbreviations for cosine and sine expressions, where the subscript $k$ defines the respective angle. The four DH-parameters are given by the relation of the two chosen coordinate systems (often placed at each joint) and is structured in the form of a DH-table.

In this paper, the right-hand-rule coordinate system convention will be used for the coordinate system and joint orientation, meaning counter-clockwise rotation is considered as positive rotation. However, the provided Comau robot interface treats clockwise rotation as positive, and the default home position of the robot is considered to be in a configuration where the forearm is pointing upwards (when $d_{4}$ is parallel to $z_{1}$, see Figure 12).

The DH parameter table can be constructed for the Comau robot, using the overview in Figure 12, and treating the presented pose as the home position. Table 2 presents the $\mathrm{DH}$ parameter table, with the corrected disparities of the Comau interface, for the full 6-DOF Comau robot without the extension arm.

Table 2: DH table for the Comau Robot (6-DOF configuration) without extension arm.

\begin{tabular}{clccc}
\hline Link $i$ & $\theta_{i}$ & $d_{i}$ & $a_{i}$ & $\alpha_{i}$ \\
\hline 1 & $-\theta_{1}$ & $d_{1}$ & $a_{1}$ & $\frac{\pi}{2}$ \\
2 & $\frac{\pi}{2}-\theta_{2}$ & 0 & $a_{2}$ & 0 \\
3 & $\theta_{3}+\frac{\pi}{2}+\theta_{2}$ & 0 & $a_{3}$ & $\frac{\pi}{2}$ \\
4 & $-\theta_{4}$ & $d_{4}$ & 0 & $-\frac{\pi}{2}$ \\
5 & $-\theta_{5}$ & 0 & 0 & $\frac{\pi}{2}$ \\
6 & $\pi-\theta_{6}$ & $d_{6}$ & 0 & 0 \\
\hline
\end{tabular}

Experiments related to VVMC and other motion compensation scenarios the Comau robot of the Motion-Lab is used to simulate a loader crane, where the wrist joints are fixed and the robot is equipped with an extension arm to hold the winch wire in place. This configuration reduces the robot to a 3 -DOF system, hence the upcoming derivation of the robot's kinematic equation will be based on the 3-DOF system. Using the geometric overview of Figure 12, the related $\mathrm{DH}$ table for 3 -DOF robot system is constructed and presented in Table 3.

Table 3: DH table for the Comau Robot (3-DOF configuration) with the extension arm.

\begin{tabular}{cllll}
\hline Link $i$ & $\theta_{i}$ & $d_{i}$ & $a_{i}$ & $\alpha_{i}$ \\
\hline 1 & $-\theta_{1}$ & $d_{1}$ & $a_{1}$ & $\frac{\pi}{2}$ \\
2 & $\frac{\pi}{2}-\theta_{2}$ & 0 & $a_{2}$ & 0 \\
3 & $\theta_{3}+\frac{\pi}{2}+\theta_{2}$ & 0 & $a_{3}$ & $\frac{\pi}{2}$ \\
4 & $\pi$ & $d_{4}+d_{6}+d_{t p}$ & 0 & 0 \\
\hline
\end{tabular}

Using Eq. (1) and the parameters described in Table 3, a complete homogeneous transformation between the coordinate frame of the robot base $\left(x_{0}, y_{0}, z_{0}\right)$ and the robot tool-point $\left(x_{t}, y_{t}, z_{t}\right)$ can be formed by.

$$
T_{t}^{0}\left(\theta_{1}, \theta_{2}, \theta_{3}\right)=\prod_{i=1}^{4} A_{i}=\left[\begin{array}{cccc}
r_{11} & r_{12} & r_{13} & x \\
r_{21} & r_{22} & r_{23} & y \\
r_{31} & r_{32} & r_{33} & z \\
0 & 0 & 0 & 1
\end{array}\right]
$$

where the first $r_{i j}$ entries of the transformation matrix $T_{t}^{0}$ contain information of the orientation of the robot's tool-point relative to the robot base, also referred to as the rotation matrix $R_{t}^{0}$.

$$
R_{t}^{0}=\left[\begin{array}{ccc}
c_{\theta_{1}} c_{\theta_{3}} & s_{\theta_{1}} & -c_{\theta_{1}} s_{\theta_{3}} \\
-c_{\theta_{3}} s_{\theta_{1}} & c_{\theta_{1}} & s_{\theta_{1}} s_{\theta_{3}} \\
s_{\theta_{3}} & 0 & c_{\theta_{3}}
\end{array}\right]=\left[\begin{array}{ccc}
r_{11} & r_{12} & r_{13} \\
r_{21} & r_{22} & r_{23} \\
r_{31} & r_{32} & r_{33}
\end{array}\right]
$$

\subsubsection{Tool-Point Position}

The first three entries of the last column of Eq. (2) describe the tool-point Cartesian position relative to the base frame of the robot. Substituting the combined length of the robot forearm and the extension arm with $L=d_{5}+d_{6}+d_{t p}$, the forward kinematic expression for the robot's tool-point position equals.

$$
p_{t}^{0}=\left[\begin{array}{c}
x_{t} \\
y_{t} \\
z_{t}
\end{array}\right]=\left[\begin{array}{c}
c_{\theta_{1}}\left(a_{1}-L s_{\theta_{3}}-a_{3} c_{\theta_{3}}+a_{2} s_{\theta_{2}}\right) \\
-s_{\theta_{1}}\left(a_{1}-L s_{\theta_{3}}-a_{3} c_{\theta_{3}}+a_{2} s_{\theta_{2}}\right) \\
d_{1}+L c_{\theta_{3}}+a_{2} c_{\theta_{2}}-a_{3} s_{\theta_{3}}
\end{array}\right]
$$

\subsubsection{Tool-Point Velocity}

The velocity components of the robot's tool-point as a function of the angular joint velocities can be found by employing the Jacobian matrix of the robot's kinematic, given by Eq. (4). 


$$
\dot{p}_{t}^{0}=\left[\begin{array}{c}
\dot{x}_{t} \\
\dot{y}_{t} \\
\dot{z}_{t}
\end{array}\right]=J\left[\begin{array}{c}
\dot{\theta}_{1} \\
\dot{\theta}_{2} \\
\dot{\theta}_{3}
\end{array}\right]
$$

where the Jacobian matrix $J$ is derived by calculating the partial derivatives of the forward kinematics equation Eq. (3).

$$
J=\left[\begin{array}{lll}
\frac{\partial x_{t}}{\partial \theta_{1}} & \frac{\partial x_{t}}{\partial \theta_{2}} & \frac{\partial x_{t}}{\partial \theta_{3}} \\
\frac{\partial y_{t}}{\partial \theta_{1}} & \frac{\partial y_{t}}{\partial \theta_{2}} & \frac{\partial y_{t}}{\partial \theta_{3}} \\
\frac{\partial z_{t}}{\partial \theta_{1}} & \frac{\partial z_{t}}{\partial \theta_{2}} & \frac{\partial z_{t}}{\partial \theta_{3}}
\end{array}\right]
$$

\subsubsection{Tool-Point Acceleration}

Introducing the derivative of the Jacobian matrix, the robot's tool-point acceleration can be expressed as a function of the joints angular velocity and acceleration.

$$
\ddot{p}_{t}^{0}=\left[\begin{array}{c}
\ddot{x}_{t} \\
\ddot{y}_{t} \\
\ddot{z}_{t}
\end{array}\right]=\dot{J}\left[\begin{array}{c}
\dot{\theta}_{1} \\
\dot{\theta}_{2} \\
\dot{\theta}_{3}
\end{array}\right]+J\left[\begin{array}{l}
\ddot{\theta}_{1} \\
\ddot{\theta}_{2} \\
\ddot{\theta}_{3}
\end{array}\right]
$$

\subsection{Inverse Kinematics}

Equations related to the inverse kinematics of the Comau robot's 3-DOF configuration is derived using the geometric decoupling approach, even though the inverse function could be derived from analyzing and rearranging Eq. (3). Considering the Comau robot in a configuration as shown in Figure 13, the tool-point coordinates can be projected onto the XY-plane of the robot's base coordinate frame $\left(x_{0}, y_{0}, z_{0}\right)$. Using this projection, the angular rotation of the robot base joint $\theta_{1}$ can be defined relative to the robot base frame coordinate by.

$$
\theta_{1}=-\operatorname{atan} 2\left(y_{t}, x_{t}\right)
$$

Opposed to the normal inverse tangent function, the atan2 function is used in the formulation of this paper. The latter function considers the sign of the vector component and places the calculated angle in the correct quadrant.

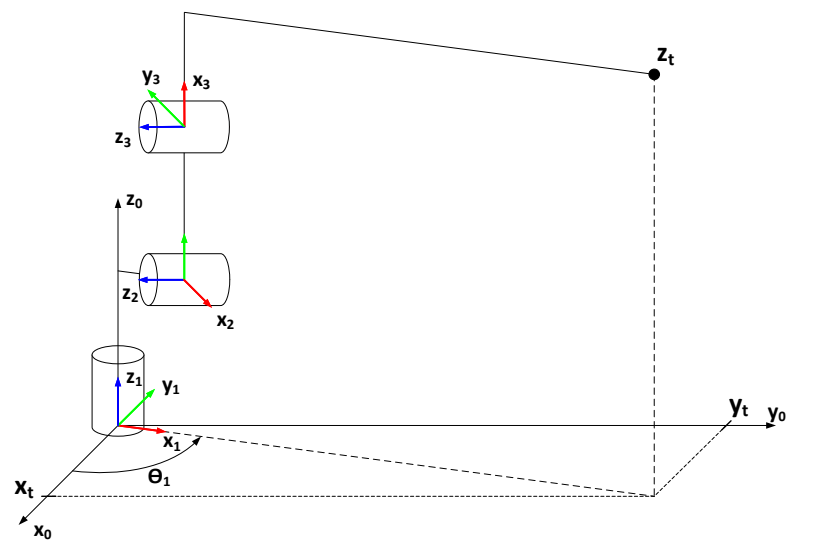

Figure 13: Geometric approach for base joint $\theta_{1}$.

Without considering the rotation of the robot base, the system can be simplified and considered as a planar two-link elbow system, where the inverse kinematics of such a configuration has two possible solutions; elbowdown and elbow-up. An illustration of the Comau robot in a planar two-link configuration is shown in Figure 14, where the link $a_{1}$ and line $B$ symbolizes the twolink elbow system.

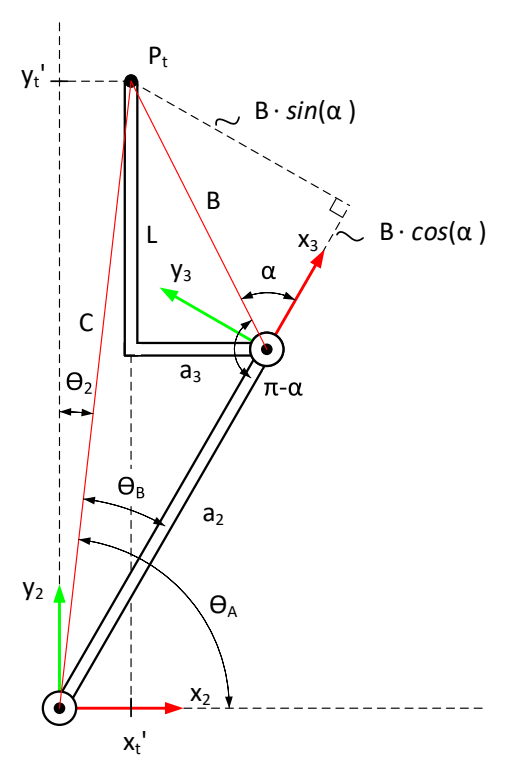

Figure 14: Geometric approach for shoulder joint $\theta_{2}$.

Line $B$ remains constant for all configurations and is defined as:

$$
B=\sqrt{a_{3}^{2}+L^{2}}
$$

The tool-point vector components given relative to the coordinate frame of the shoulder joint $\left(x_{2}, y_{2}, z_{2}\right)$ 
are indicated by $\left(x_{t}^{\prime}, y_{t}^{\prime}\right)$. The value of these components can be calculated by the product of the inverse transformation matrix $A_{1}$ and the tool-point position given relative to the base frame coordinate.

$$
\left[\begin{array}{c}
x_{t}{ }^{\prime} \\
y_{t}{ }^{\prime} \\
z_{t}{ }^{\prime} \\
1
\end{array}\right]=\left(A_{1}\right)^{-1}\left[\begin{array}{c}
x_{t} \\
y_{t} \\
z_{t} \\
1
\end{array}\right]
$$

Eq. (10) is derived by introducing the law of cosines for the triangle $a_{2} B C$, where the length of line $C$ is defined by Eq. (11).

$$
\begin{aligned}
C^{2} & ={a_{2}}^{2}+B^{2}-2 a_{2} B \cos (\pi-\alpha) \\
C & =\sqrt{\left(x_{t}^{\prime}\right)^{2}+\left(y_{t}^{\prime}\right)^{2}}
\end{aligned}
$$

Using the properties of the unit circle $(\cos (\pi-\alpha)=$ $-\cos \alpha)$ Eq. (10) can be rewritten. Here $D$ is introduced and defined equal to $\cos \alpha$ for easier notation.

$$
\cos \alpha=\frac{C^{2}-a_{2}{ }^{2}-B^{2}}{2 a_{2} B}:=D
$$

Instead of directly deriving the angle $\alpha$ from Eq. (12), the Pythagorean identity is introduced.

$$
\begin{aligned}
\sin ^{2} \alpha+\cos ^{2} \alpha & =1 \\
\Rightarrow \sin \alpha & = \pm \sqrt{1-D^{2}}
\end{aligned}
$$

An expression for the angle $\alpha$ can now be defined as a combination of the Pythagorean identity Eq. (13), and the previous derived Eq. (12).

$$
\begin{aligned}
\alpha & =\operatorname{atan} 2(\sin \alpha, D) \\
& =\operatorname{atan} 2\left( \pm \sqrt{1-D^{2}}, D\right)
\end{aligned}
$$

Expressing the angle $\alpha$ as defined by (15) introduces the possibility to select the elbow configuration based on the two solutions of the square root. An elbow-down or an elbow-up configuration is selected by choosing a negative or a positive sign, respectively (Spong et al., 2005).

The two assisting angles $\theta_{A}$ and $\theta_{B}$ are defined by Eq. (16) and Eq. (17), and the angle for the robot's shoulder joint $\theta_{2}$ is calculated by Eq. (18)

$$
\begin{aligned}
\theta_{A} & =\operatorname{atan} 2\left(y_{t}{ }^{\prime}, x_{t}{ }^{\prime}\right) \\
\theta_{B} & =\operatorname{atan} 2\left(B \sin \alpha, a_{2}+B \cos \alpha\right) \\
\theta_{2} & =\frac{\pi}{2}-\left(\theta_{A}-\theta_{B}\right)
\end{aligned}
$$

The robot's elbow joint $\theta_{3}$ is derived from the same geometric approach of the planar two-link elbow system. Figure 15, shows the two-link elbow system with a small angular offset in the elbow joint.

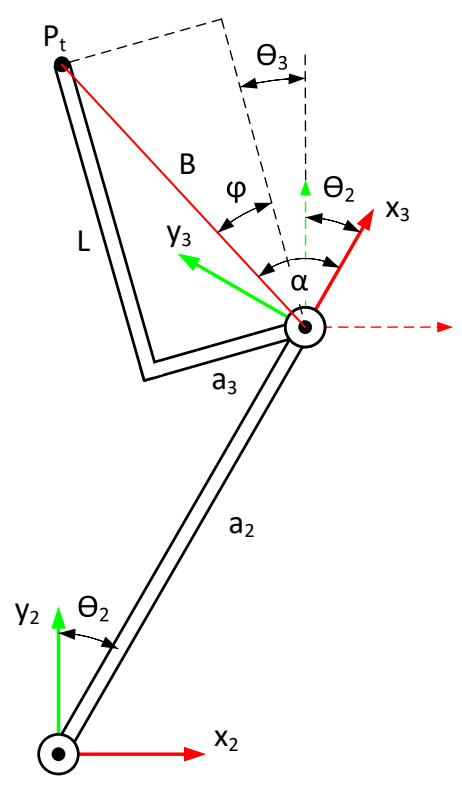

Figure 15: Geometric approach for elbow joint $\theta_{3}$.

Analyzing the geometries of Figure 15, the elbow joint $\theta_{3}$ can be defined as Eq. (20), where $\alpha$ and $\theta_{2}$ are found by Eq. (15) and Eq. (18), and the constant angle $\varphi$ is equal to Eq. (19).

$$
\begin{aligned}
\varphi & =\operatorname{atan} 2\left(a_{3}, L\right) \\
\theta_{3} & =\alpha-\varphi-\theta_{2}
\end{aligned}
$$

\subsubsection{Joint Angular Velocity}

Introducing the inverse of the previously defined Jacobian matrix of Eq. (5), the angular velocities of the robot joints are derived as the product of the inverse Jacobian and the tool-point velocity.

$$
\begin{gathered}
\dot{\theta}=J^{-1} \dot{p}_{t}^{0} \\
\Rightarrow\left[\begin{array}{c}
\dot{\theta}_{1} \\
\dot{\theta}_{2} \\
\dot{\theta}_{3}
\end{array}\right]=J^{-1}\left[\begin{array}{l}
\dot{x}_{t} \\
\dot{y}_{t} \\
\dot{z}_{t}
\end{array}\right]
\end{gathered}
$$

\subsubsection{Joint Angular Acceleration}

Rearranging Eq. (6) yields an expression for the angular acceleration of the robot joints, which is dependent on the inverse Jacobian matrix, tool-point acceleration and the derivative of the Jacobian matrix. 


$$
\begin{gathered}
\ddot{\theta}=J^{-1}\left(\ddot{p}_{t}^{0}-\dot{j} \dot{\theta}\right) \\
\Rightarrow\left[\begin{array}{l}
\ddot{\theta}_{1} \\
\ddot{\theta}_{2} \\
\ddot{\theta}_{3}
\end{array}\right]=J^{-1}\left(\left[\begin{array}{l}
\ddot{x}_{t} \\
\ddot{y}_{t} \\
\ddot{z}_{t}
\end{array}\right]-\dot{J}\left[\begin{array}{l}
\dot{\theta}_{1} \\
\dot{\theta}_{2} \\
\dot{\theta}_{3}
\end{array}\right]\right)
\end{gathered}
$$

\section{Suspended Load Motion}

Problems concerning compensation and motion stabilization of load handling operations require an understanding of the motion and general behavior of a suspended load. This section will derive the governing equations of motion for a 3-dimensional (3D) suspended load, which are essential for further development of motion compensation solutions.

An illustration of a 3D suspended load system depicted in Figure 16, where the suspended load $p_{p}$ with mass $m_{p}$ is connected to the tool-point $p_{t}$ via a wire of length $L_{w}$.

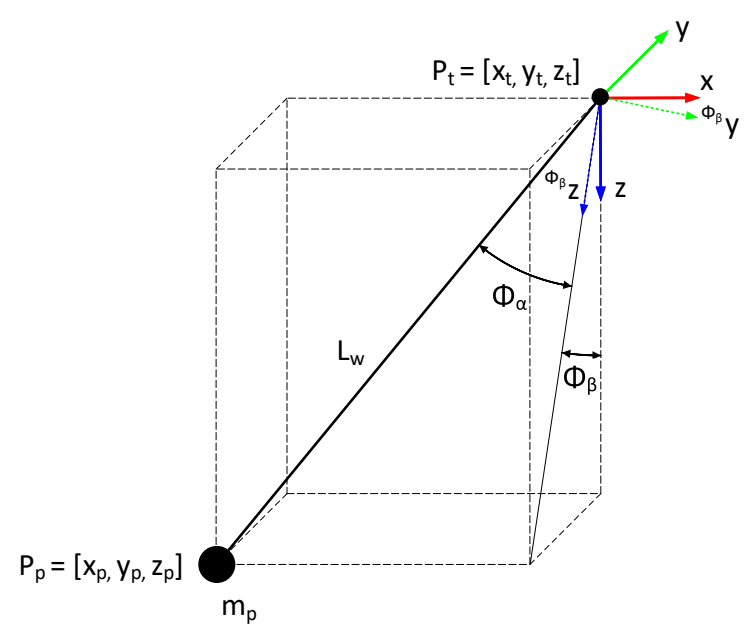

Figure 16: Illustration of a 3D suspend load represented by Euler-angles.

The orientation of the suspended load is represented as a set of Euler-angles (Gustafsson, 1993), when in motion the load will initiate an angle $\phi_{\beta}$ around the $x$-axis and an angle $\phi_{\alpha}$ around the ${ }^{\phi_{\beta}} y$-axis, where ${ }^{\phi_{\beta}} y$ and ${ }^{\phi_{\beta}} z$ emphasize the coordinate frame created by the rotation $\phi_{\beta}$ around the $x$-axis.

Derivation of the governing equations of motion follows a few assumptions for the suspended load system. The payload is assumed to be a point mass, the wire is assumed to be a massless rigid rod with deflection and elongation neglected, and frictional elements between the wire and the tool-point are also neglected.

\subsection{Payload Position}

Employing the Euler-angle representation of Figure 16, the position of the suspended load can be derived as given by Eq. (23).

$$
p_{p}=p_{t}+L_{w}\left[\begin{array}{c}
-s_{\phi_{\alpha}} \\
c_{\phi_{\alpha}} s_{\phi_{\beta}} \\
c_{\phi_{\alpha}} c_{\phi_{\beta}}
\end{array}\right]
$$

\subsection{Payload Velocity}

The velocity of the suspended load is found by the timederivative of Eq. (23) and is given by Eq. (24).

$$
\begin{aligned}
& \dot{p}_{p}=\dot{p}_{t}+L_{w} {\left[\begin{array}{c}
-\dot{\phi}_{\alpha} c_{\phi_{\alpha}} \\
-\dot{\phi}_{\alpha} s_{\phi_{\alpha}} s_{\phi_{\beta}}+\dot{\phi}_{\beta} c_{\phi_{\alpha}} c_{\phi_{\beta}} \\
-\dot{\phi}_{\alpha} s_{\phi_{\alpha}} c_{\phi_{\beta}}-\dot{\phi}_{\beta} c_{\phi_{\alpha}} s_{\phi_{\beta}}
\end{array}\right] } \\
&+\dot{L}_{w}\left[\begin{array}{c}
-s_{\phi_{\alpha}} \\
c_{\phi_{\alpha}} s_{\phi_{\beta}} \\
c_{\phi_{\alpha}} c_{\phi_{\beta}}
\end{array}\right]
\end{aligned}
$$

\subsection{Equation of Motion}

The equations of motion describing the suspended load's dynamics are derived using the Euler-Lagrange approach. The Lagrangian which describes the difference between a system's kinetic and potential energy is given by Eq. (25).

$$
\mathcal{L}=\mathcal{K}-\mathcal{P}
$$

where the kinetic energy $\mathcal{K}$ for the suspended load equals Eq. (26)

$$
\mathcal{K}=\frac{1}{2} m_{p} \dot{p}_{p}^{T} \dot{p}_{p}
$$

and the potential energy $\mathcal{P}$ for the load is found by Eq. (27)

$$
\mathcal{P}=m_{p} g L_{w}\left(1-c_{\phi_{\alpha}} c_{\phi_{\beta}}\right)
$$

The Euler-Lagrange equation is defined as given by Eq. (28)

$$
\frac{d}{d t} \frac{\partial \mathcal{L}}{\partial \dot{q}_{k}}-\frac{\partial \mathcal{L}}{\partial q_{k}}=0
$$

where $q_{k}$ is the generalized coordinate vector consisting of the Euler-angles $\phi_{\alpha}$ and $\phi_{\beta}$. Solving Eq. (28) gives a coupled pair of second order differential equations describing the dynamics of the $3 \mathrm{D}$ suspended load. 


$$
\begin{aligned}
L_{w} \ddot{\phi}_{\alpha}= & \ddot{x}_{t} c_{\phi_{\alpha}}+\ddot{y}_{t} s_{\phi_{\alpha}} s_{\phi_{\beta}}-\ddot{z}_{t} s_{\phi_{\alpha}} c_{\phi_{\beta}} \\
& -g s_{\phi_{\alpha}} c_{\phi_{\beta}}-2 \dot{L}_{w} \dot{\phi}_{\alpha}-L_{w} \dot{\phi}_{\beta}^{2} s_{\phi_{\alpha}} c_{\phi_{\alpha}} \\
L_{w} c_{\phi_{\alpha}} \ddot{\phi}_{\beta}= & -\ddot{y}_{t} c_{\phi_{\beta}}-\ddot{z}_{t} s_{\phi_{\beta}}-g s_{\phi_{\beta}} \\
& -2 \dot{L}_{w} \dot{\phi}_{\beta} c_{\phi_{\alpha}}+2 L_{w} \dot{\phi}_{\alpha} \dot{\phi}_{\beta} s_{\phi_{\alpha}}
\end{aligned}
$$

\section{Full Motion System Kinematics}

The experimental setup of the Motion-Lab uses the two SPs to simulate the motion of two floating vessels. With the Comau robot installed on top of the largest SP, the simulated 6 -DOF wave motion will introduce motion relative to the base of the robot, leading to the robot's tool-point and the suspended load to be influenced by both wave motion and actuation of the robot joints.

In VVMC experiments, the load handling equipment tries to compensate for the motion of both vessels, with the aim of stabilizing the hanging load relative to the secondary vessel. Hence, an investigation of the related kinematics and relative motion is essential for understanding the behavior of the full motion system.

An illustration of the experimental setup is depicted in Figure 17, here both of the SPs can be seen together with the Comau robot and the suspended load. The overview shows the notation of the assigned coordinate systems and homogeneous transformation between them. A world coordinate is added which acts as a reference for the systems in motion. Detailed description for the notations of the coordinate frame are given by Table 4 .

Table 4: Coordinate system annotations used for the full motion system kinematics.

\begin{tabular}{ll}
\hline Notation & Description \\
\hline$\left\{b_{g}\right\}$ & World Coordinate \\
$\left\{b_{n_{1}}\right\}$ & E-Motion 8000 (Neutral) \\
$\left\{b_{b_{1}}\right\}$ & E-Motion 8000 (Body-fixed) \\
$\left\{b_{n_{2}}\right\}$ & E-Motion 1500 (Neutral) \\
$\left\{b_{b_{2}}\right\}$ & E-Motion 1500 (Body-fixed) \\
$\left\{b_{r}\right\}$ & Comau Robot (Base) \\
$\left\{b_{t}\right\}$ & Comau Robot (Tool-point) \\
$\left\{b_{p}\right\}$ & Suspended Payload \\
\hline
\end{tabular}

As illustrated by the overview (Figure 17) both of the SPs are assigned with two coordinate systems, a neutral-frame, and a body-fixed frame. The former describes the neutral position and orientation of the

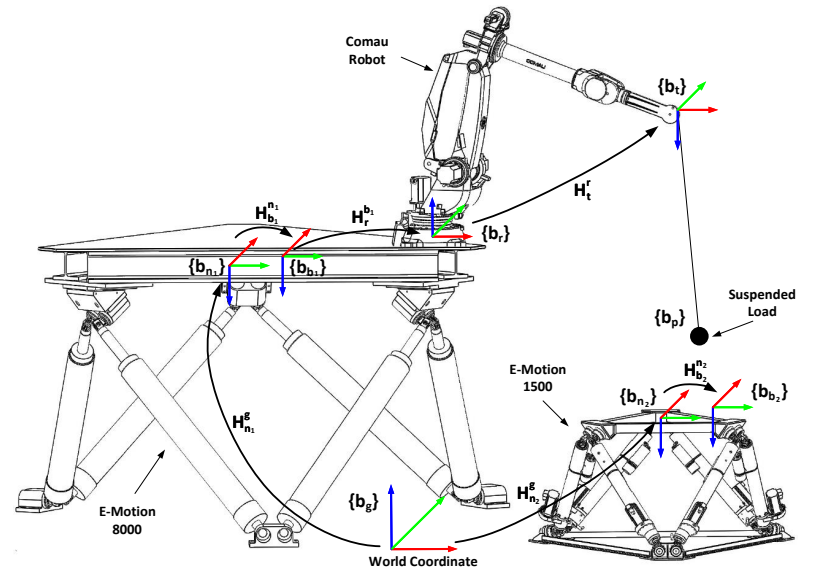

Figure 17: An overview of the experimental motion system setup, with body-fixed coordinate systems and transformations.

platforms when the platforms are not exposed to waveinduced motion, and the body-fixed frame describes the position and orientation for when the platforms are in motion. This notation is introduced to easier describe the homogeneous transformation of the platforms, where the platform motion can be described relative to the neutral-frame.

Homogeneous transformations are used to describe the relative motion of equipment, where the world coordinate $\left\{b_{g}\right\}$ acts as a general reference to the different frames shown in Figure 17. The primary transformations are depicted in the overview, where the subscripts and superscripts refer to the focus frame and relative frame, respectively. The homogeneous transformations which remain constant during the motion simulations are obtainable through calibration as described in Heng and Tørdal (2017), which present an approach to calibrate the fixed transformations. The resulting calibration data describing the homogeneous calibration matrices are loaded into the MATLAB workspace by executing the following command load('motionlab/calib.mat'), where the calibration data is found in the loaded calib MATLAB structure. Table 5 lists the calibration matrices presented in this paper together with their respective naming in the MATLAB structure.

\subsection{Stewart Platform Kinematics}

The motion generated by the SP aims to simulate a vessel experiencing 6-DOF wave-induced motion. Figure 18 shows the general definition of the axes and orientation related to the 6-DOF. Sway $(x)$, surge $(y)$ and heave $(z)$ are notations for the translations along the axes, and roll $(\phi)$, pitch $(\theta)$ and yaw $(\psi)$ are the 
Table 5: Fixed homogeneous transformations obtainable from the calibration MATLAB structure found from loading the calibration file.

\begin{tabular}{ll}
\hline Notation & MATLAB Structure \\
\hline$H_{n_{1}}^{g}$ & calib.WORLD_TO_EM8000.H \\
$H_{n_{2}}^{g}$ & calib.WORLD_TO_EM1500.H \\
$H_{b_{r}}^{b_{1}}$ & calib.EM8000_TO_COMAU.H \\
\hline
\end{tabular}

related rotational angles.

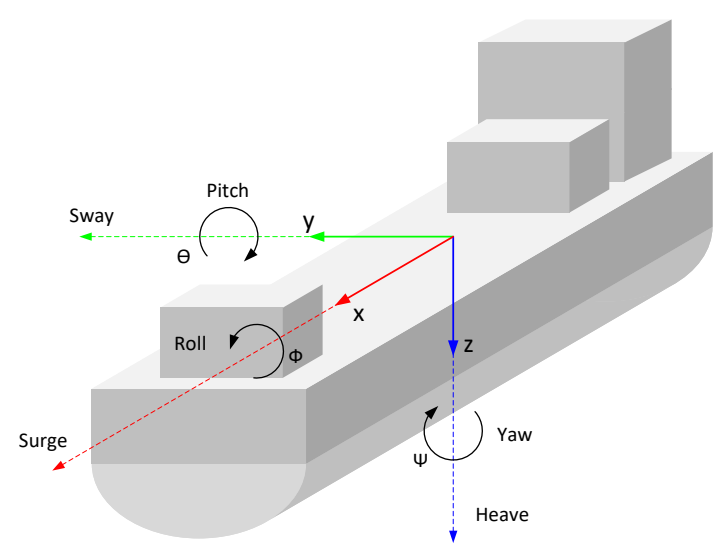

Figure 18: Definition of axes and orientation for vessels out at sea.

The notation of Figure 18 is used to describe the relative motion for the configuration in Figure 17. A translation-vector $p_{b}^{n}$ given by Eq. (31), can be introduced to define the position of the body-fixed frame $\left\{b_{b}\right\}$ relative to the neutral frame $\left\{b_{n}\right\}$ using the surge, sway and heave components.

$$
p_{b}^{n}=\left[\begin{array}{l}
x \\
y \\
z
\end{array}\right]
$$

A vector $\delta$ is used to describe the roll, pitch and yaw components of the SP.

$$
\delta=\left[\begin{array}{l}
\phi \\
\theta \\
\psi
\end{array}\right]
$$

Using the roll, pitch and yaw components, a rotation matrix $R_{n}^{b}$ describing the relative orientation of frame $\left\{b_{b}\right\}$ given in $\left\{b_{n}\right\}$ can be derived as a successive sequence of rotations, shown by Eq. (33).

$$
R_{b}^{n}(\delta)=R_{x}(\psi) R_{y}(\theta) R_{z}(\phi)
$$

where:

$$
\begin{aligned}
R_{x}(\psi)= & {\left[\begin{array}{ccc}
1 & 0 & 0 \\
0 & c_{\psi} & -s_{\psi} \\
0 & s_{\psi} & c_{\psi}
\end{array}\right] } \\
R_{y}(\theta)= & {\left[\begin{array}{ccc}
c_{\theta} & 0 & s_{\theta} \\
0 & 1 & 0 \\
-s_{\theta} & 0 & c_{\theta}
\end{array}\right] } \\
R_{z}(\phi)= & {\left[\begin{array}{ccc}
c_{\phi} & -s_{\phi} & 0 \\
s_{\phi} & c_{\phi} & 0 \\
0 & 0 & 1
\end{array}\right] }
\end{aligned}
$$

The sway, surge and heave vector $p_{b}^{n}$ (Eq. (31)) and the roll, pitch and yaw vector $\delta$ (Eq. (32)) are collected, allowing the pose components of the SP to be fully described by a combined vector $\eta$, given by Eq. (37).

$$
\eta=\left[\begin{array}{c}
p_{n}^{b} \\
\delta
\end{array}\right]=\left[\begin{array}{c}
x \\
y \\
z \\
\phi \\
\theta \\
\psi
\end{array}\right]
$$

$\eta$ which contains the components describing the relative position and rotation of the body-fixed frame $\left\{b_{b}\right\}$ given in $\left\{b_{n}\right\}$, is together with the respective time derivative $\dot{\eta}$ (velocity) and second time derivative $\ddot{\eta}$ (acceleration) characterized as the governing parameters for the Stewart Platform motion.

\subsubsection{Stewart Platform Pose}

Continuing using the E-Motion 8000 as an example, the relative pose of the SP can be fully described as the homogeneous transformation matrix $H_{b}^{n}$, given by Eq. (38).

$$
H_{b}^{n}=\left[\begin{array}{cc}
R_{b}^{n}(\delta) & p_{b}^{n} \\
0 & 1
\end{array}\right]
$$

Utilizing the obtained calibration data of $H_{n}^{g}$, the position and orientation of the Stewart platform $\left\{b_{b}\right\}$ relative to the world coordinate frame $\left\{b_{g}\right\}$ can be described as.

$$
H_{b}^{g}=H_{n}^{g} H_{b}^{n}
$$

\subsubsection{Stewart Platform Velocity}

The relative motion generated by the SPs introduces both translational and rotational velocities to the equipment installed on the platforms. Hence the desire for expressing the translational and angular velocity relative to the platform's body-fixed frame $\left\{b_{b}\right\}$.

The time derivative of the combined vector $\eta$ (given by Eq. (37)), equals to. 


$$
\dot{\eta}=\left[\begin{array}{c}
\dot{p}_{b}^{n} \\
\dot{\delta}
\end{array}\right]
$$

A vector $\omega_{n, b}^{b}$ is introduced, which describes the body-fixed angular velocity of the SP expressed in $\left\{b_{b}\right\}$. The relation between the angular velocity vector $\omega_{n, b}^{b}$ and governing vector $\dot{\delta}$ is described by Eq. (41) (Fossen, 2011).

$$
\dot{\eta}=J_{s} v
$$

where $v$ is defined as.

$$
v=\left[\begin{array}{c}
\dot{p}_{b}^{n} \\
\omega_{n, b}^{b}
\end{array}\right]
$$

and $J_{s}$ is referred to as the ship Jacobian, given by Eq. (43).

$$
J_{s}=\left[\begin{array}{cc}
I & 0 \\
0 & T(\delta)
\end{array}\right]
$$

$I$ is defined as the identity matrix, and $T(\delta)$ is a transformation matrix, defined by Eq. (44).

$$
T(\delta)=\left[\begin{array}{ccc}
\frac{c_{\psi}}{c_{\theta}} & -\frac{s_{\psi}}{c_{\theta}} & 0 \\
s_{\psi} & c_{\psi} & 0 \\
-\frac{s_{\theta} c_{\psi}}{c_{\theta}} & \frac{s_{\theta} s_{\psi}}{c_{\theta}} & 1
\end{array}\right]
$$

Using the definitions of Eq. (42) to Eq. (44), the expression of Eq. (41) can be rearranged and solved for vector $v$.

$$
v=\left[\begin{array}{c}
\dot{p}_{b}^{n} \\
\omega_{n, b}^{b}
\end{array}\right]=J_{s}^{-1} \dot{\eta}
$$

The notion of skew matrices is introduced, where the skew matrix for an arbitrary vector $\beta=\left[\beta_{x}, \beta_{y}, \beta_{z}\right]^{T}$ is given by.

$$
S(\beta)=\left[\begin{array}{ccc}
0 & -\beta_{z} & \beta_{y} \\
\beta_{z} & 0 & -\beta_{x} \\
-\beta_{y} & \beta_{x} & 0
\end{array}\right]
$$

The angular velocity of the SP can now be expressed as the time derivative of the rotational matrix $R_{b}^{n}$, which is derived using the skew matrix of the bodyfixed velocity vector $\omega_{n, b}^{b}$.

$$
\dot{R}_{b}^{n}=R_{b}^{n} S\left(\omega_{n, b}^{b}\right)
$$

\subsubsection{Stewart Platform Acceleration}

The translational and angular acceleration generated by the motion of the SP is defined by Eq. (48).

$$
\ddot{\eta}=\left[\begin{array}{c}
\ddot{p}_{b}^{n} \\
\ddot{\delta}
\end{array}\right]
$$

Acceleration relative to the body-fixed frame $\left\{b_{b}\right\}$ is derived by following the same approach as for the velocity.

The time derivative of Eq. (41) equals:

$$
\ddot{\eta}=\dot{J}_{s} v+J_{s} \dot{v}
$$

Eq. (49) is rearranged and solved for $\dot{v}$.

$$
\dot{v}=\left[\begin{array}{c}
\dot{p}_{b}^{n} \\
\dot{\omega}_{n, b}^{b}
\end{array}\right]=J_{s}^{-1}\left(\ddot{\eta}-\dot{J}_{s} \dot{\eta}\right)
$$

The rotational acceleration of the $\mathrm{SP}$ is defined as the time derivative of Eq. (47), given by Eq. (51)

$$
\begin{aligned}
\ddot{R}_{b}^{n} & =\dot{R}_{b}^{n} S\left(\omega_{n, b}^{b}\right)+R_{b}^{n} S\left(\dot{\omega}_{n, b}^{b}\right) \\
& =R_{b}^{n} S\left(\omega_{n, b}^{b}\right) S\left(\omega_{n, b}^{b}\right)+R_{b}^{n} S\left(\dot{\omega}_{n, b}^{b}\right)
\end{aligned}
$$

\subsection{Updated Robot Tool-Point Motion}

The equations describing the Comau robot kinematics (Section 4) are derived relative to the internal frame coordinate, which corresponds to the base-frame of the robot $\left\{b_{r}\right\}$. The wave motion simulated by the EM8000 will introduce a motion to the robot base, meaning that the equations describing the robot's tool-point needs to be updated for the additional relative motion.

The available calibration matrices of Table 5, presents the homogeneous transformation matrix $H_{r}^{b}$ describing the pose of the robot base frame $\left\{b_{r}\right\}$ relative to the body-fixed frame $\left\{b_{b_{1}}\right\}$ of the EM- 8000 . This transformation can be expressed by a rotation matrix and a translation vector as shown by Eq. (52).

$$
H_{r}^{b_{1}}=\left[\begin{array}{cc}
R_{r}^{b_{1}} & p_{r}^{b_{1}} \\
0 & 1
\end{array}\right]
$$

In the same manner, the calibrated transformation matrix $H_{n_{1}}^{g}$ describing the position and orientation of the EM-8000's neutral-frame $\left\{b_{n_{1}}\right\}$ given in the world coordinate frame $\left\{b_{g}\right\}$, can be expressed as Eq. (53).

$$
H_{n_{1}}^{g}=\left[\begin{array}{cc}
R_{n_{1}}^{g} & p_{n_{1}}^{g} \\
0 & 1
\end{array}\right]
$$

\subsubsection{Robot Tool-Point Position}

The robot tool-point position relative to the neutralframe $\left\{b_{n_{1}}\right\}$ of the EM8000, can be formulated as shown by Eq. (54).

$$
p_{t}^{n_{1}}=p_{b_{1}}^{n_{1}}+R_{b_{1}}^{n_{1}}\left(p_{r}^{b_{1}}+R_{r}^{b_{1}} p_{t}^{r}\right)
$$

where $p_{b_{1}}^{n_{1}}$ and $R_{b_{1}}^{n_{1}}$ are the position and orientation of the EM-8000, given by Eq. (31) and Eq. (33) respectively. $p_{t}^{r}$ is defined as the robot's tool-point position 
relative to the base-frame $\left\{b_{r}\right\}$, which equals the previously derived vector describing the tool-point position relative to the internal frame $p_{t}^{0}$ (Eq. (3)).

Using Eq. (53) and Eq. (54), the robot's tool-point relative to the world coordinate frame is given by Eq. (55)

$$
p_{t}^{g}=p_{n_{1}}^{g}+R_{n_{1}}^{g} p_{t}^{n_{1}}
$$

\subsubsection{Robot Tool-Point Velocity}

The expression for the velocity of the robot's tool-point is found from the time-derivative of Eq. (54). The calibrated transformation matrix $H_{n}^{g}$ is a constant relation, hence the tool-point velocity expressed relative to the world coordinate will equal the expression given relative to the neutral-frame.

$$
\begin{aligned}
\dot{p}_{t}^{g}=\dot{p}_{t}^{n_{1}} & =\dot{p}_{b_{1}}^{n_{1}}+\dot{R}_{b_{1}}^{n_{1}}\left(p_{r}^{b_{1}}+R_{r}^{b_{1}} p_{t}^{r}\right) \\
& +R_{b_{1}}^{n_{1}}\left(R_{r}^{b_{1}} \dot{p}_{t}^{r}\right)
\end{aligned}
$$

where $\dot{R}_{b_{1}}^{n_{1}}$ is given by Eq. (47), and $\dot{p}_{t}^{r}$ equals the previously derived Eq. (4).

\subsubsection{Robot Tool-Point Acceleration}

The updated equation for the acceleration of the robot's tool-point is given as the time-derivative of Eq. (56).

$$
\begin{aligned}
\ddot{p}_{t}^{g}=\ddot{p}_{t}^{n_{1}}= & \ddot{p}_{b_{1}}^{n_{1}}+\ddot{R}_{b_{1}}^{n_{1}}\left(p_{r}^{b_{1}}+R_{r}^{b_{1}} p_{t}^{r}\right) \\
& +2 \dot{R}_{b_{1}}^{n_{1}}\left(R_{r}^{b_{1}} \dot{p}_{t}^{r}\right)+R_{b_{1}}^{n_{1}}\left(R_{r}^{b_{1}} \ddot{p}_{t}^{r}\right)
\end{aligned}
$$

where the rotational acceleration of the EM- 8000 $\ddot{R}_{b_{1}}^{n_{1}}$, is given by Eq. (51), and the tool-point acceleration given relative to the robot base-frame $\ddot{p}_{t}^{r}$, is derived by Eq. (6).

\subsection{Updated Suspended Load Motion}

In the same manner, as the motion of the SP introduces a relative motion to the robot base, the suspended load will experience relative motion to the world coordinate. A set of expressions describing the updated equations for the motion of the suspended load can be derived by combing the earlier derived expressions (Sec. 5) with the updated expressions describing the motion of robot's tool-point (Sec. 6.2).

\subsubsection{Suspended Load Position}

The updated equation describing the position of the suspended load relative to the world coordinate frame is given by Eq. (58).

$$
p_{p}^{g}=p_{t}^{g}+L_{w}\left[\begin{array}{c}
-s_{\phi_{\alpha}} \\
c_{\phi_{\alpha}} s_{\phi_{\beta}} \\
c_{\phi_{\alpha}} c_{\phi_{\beta}}
\end{array}\right]
$$

where $p_{t}^{g}=\left[x_{t}^{g}, y_{t}^{g}, z_{t}^{g}\right]$ describes the components of tool-point's position vector given in the world coordinate frame.

\subsubsection{Suspended Load Velocity}

The updated expression for the velocity of the suspended load is derived from the time derivative of Eq. (58) and is given by Eq. (59)

$$
\begin{aligned}
\dot{p}_{p}^{g}=\dot{p}_{t}^{g}+L_{w}\left[\begin{array}{c}
-\dot{\phi}_{\alpha} c_{\phi_{\alpha}} \\
-\dot{\phi}_{\alpha} s_{\phi_{\alpha}} s_{\phi_{\beta}}+\dot{\phi}_{\beta} c_{\phi_{\alpha}} c_{\phi_{\beta}} \\
-\dot{\phi}_{\alpha} s_{\phi_{\alpha}} c_{\phi_{\beta}}-\dot{\phi}_{\beta} c_{\phi_{\alpha}} s_{\phi_{\beta}}
\end{array}\right] \\
+\dot{L}_{w}\left[\begin{array}{c}
-s_{\phi_{\alpha}} \\
c_{\phi_{\alpha}} s_{\phi_{\beta}} \\
c_{\phi_{\alpha}} c_{\phi_{\beta}}
\end{array}\right]
\end{aligned}
$$

where $\dot{p}_{t}^{g}$ is defined as the velocity vector of the robot's tool-point relative to the world coordinate frame.

\subsubsection{Suspended Load Equation of Motion}

The coupled equations describing the dynamics of the suspended load (previously given by Eq. (60) and Eq. (61)) can be updated for platform motion by employing the components of the new expression for the tool-point acceleration vector $\left(\ddot{p}_{t}^{g}=\left[\ddot{x}_{t}^{g}, \ddot{y}_{t}^{g}, \ddot{z}_{t}^{g}\right]\right.$ given by Eq. (57)).

$$
\begin{aligned}
L_{w} \ddot{\phi}_{\alpha}= & \ddot{x}_{t}^{g} c_{\phi_{\alpha}}+\ddot{y}_{t}^{g} s_{\phi_{\alpha}} s_{\phi_{\beta}}-\ddot{z}_{t}^{g} s_{\phi_{\alpha}} c_{\phi_{\beta}} \\
& -g s_{\phi_{\alpha}} c_{\phi_{\beta}}-2 \dot{L}_{w} \dot{\phi}_{\alpha}-L_{w} \dot{\phi}_{\beta}^{2} s_{\phi_{\alpha}} c_{\phi_{\alpha}} \\
L_{w} c_{\phi_{\alpha}} \ddot{\phi}_{\beta}= & -\ddot{y}_{t}^{g} c_{\phi_{\beta}}-\ddot{z}_{t}^{g} s_{\phi_{\beta}}-g s_{\phi_{\beta}} \\
& -2 \dot{L}_{w} \dot{\phi}_{\beta} c_{\phi_{\alpha}}+2 L_{w} \dot{\phi}_{\alpha} \dot{\phi}_{\beta} s_{\phi_{\alpha}}
\end{aligned}
$$

\section{Experiments}

The Norwegian Motion-Laboratory is a unique testing facility available for researchers, students and external partners to conduct related experiments and simulations. The presented configuration and equipment of the Motion-Lab have already been utilized in several successful experiments. Several projects with external industry partners have been completed, many of which have resulted in internal reports for the partners. The open publications from the laboratory are summarized below. 
Luxcey et al. (2014) investigated the effects of waveinduced skid motion on the launch of free-fall lifeboats. In this research, the Motion-Lab was used to simulate the wave motion and drop-test experiments were conducted in the laboratory.

Kjelland (2016) used the Motion-Lab in his Ph.D. work focusing on motion compensation and load transfer from a bottom-fixed hydraulic crane to a moving platform (the large Stewart platform).

Tørdal et al. (2017a) used the available Comau robot to investigate the implementation of the inverse kinematic solution using Conformal Geometric Algebra (CGA).

CGA was also applied as a tool to calibrate the robotic equipment of the Motion-Lab in Heng and Tørdal (2017). Here a high precision Laser tracker was utilized to obtain positional data samples of the EM8000, and the CGA approach was benchmarked against conventional methods.

Tørdal et al. (2016) used the Motion-Lab to investigate the kinematics related to 6 -DOF VVMC between two floating vessels, and to test the sensor accuracy and performance of the installed MRUs in a medium-scale 1-DOF VVMC scenario.

In Tørdal and Hovland (2017a) a developed control algorithm for VVMC was implemented and experimentally tested in the Motion-Lab. Here the industrial robot is used to simulate a load handling equipment, and a control algorithm is implemented to keep the end-effector in a constant height above the secondary vessel. The installed MRUs are used to obtain realistic measurements of the SPs motion.

A novel approach using sensor fusion to track the relative motion of two floating vessels was carried out by Tørdal and Hovland (2017b). The method was based on utilizing the SPs of the Motion-Lab to simulate two floating vessels, and by using the available MRUs combined with a vision system to estimate the relative motion of two moving offshore vessels. The conducted experiments used an Aruco marker to provide measurements between the body-fixed coordinate of the MRUs, where the developed sensor fusion algorithm demonstrated the ability of self-calibration and real-time motion tracking.

Tørdal et al. (2017b) investigate the ability of the Leica AT960 laser tracker to detect the relative position and orientation between the two moving SPs, as a solution to the 6-DOF VVMC problem. Here several experiments are carried out to validate and verify the effectiveness of using the laser tracker to solve the relative pose detection problem.

\section{Conclusion}

In this paper, the Norwegian Motion Laboratory is presented and the accompanying infrastructure, configuration, and functionality are briefly covered. The installed equipment enables researchers to conduct various experiments related to different motion scenarios such as motion-compensation tasks, accuracy performance evaluation of measurements systems (LIDARs, MRUs), playback of 6-DOF time-series (wave-induced vessel motions), dynamic drop test, etc. In addition to the description of the facility, this paper aims towards giving new users of the Motion-Lab an introduction to the functionality and how to interact with the infrastructure.

A description of the current communication and connection configuration for the installed equipment is presented. The developed control interface, which acts as the main interaction for the functionality of the Motion-Lab is introduced

Detailed descriptions of the kinematics related to the industrial robot, suspended load, and motion platforms are covered. In addition, the updated expressions describing the relative motion experienced by the robot and the load due to induced motion of the platform are presented.

Technical information and additional specification of the installed equipment are available in the appendix. Here, the developed MATLAB library is available, containing useful functions related to the kinematics of the Motion-Lab.

\section{Acknowledgments}

The work presented in this paper has received funding from the Norwegian Research Council, SFI Offshore Mechatronics, project number 237896.

\section{References}

Beckhoff. Basic CPU Module. URL: https://www . beckhoff.com/CX2040/, 2018a. Visited on Jul. 16, 2018 .

Beckhoff. Beckhoff CX2040. URL: https: //www. beckhoff.com/english.asp?embedded_ pc/cx2040.htm, 2018b. Visited on Aug. 17, 2018.

Beckhoff. Beckhoff Information System ADS. URL: https://infosys.beckhoff.com/ english.php?content=. . / content/1033/tc3_ c/9007200630561931.html\&id=, 2018. Visited on Jul. 16, 2018. 


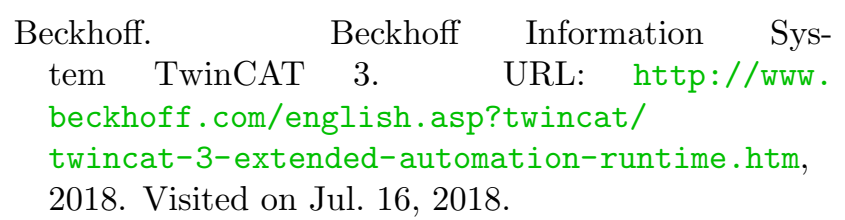

Beckhoff. Beckhoff Information System TwinCAT 3 XAR. URL: http://www. beckhoff.com/english.asp?twincat/ twincat-3-extended-automation-runtime. htm?id=1893323218933308, 2018. Visited on Jul. $16,2018$.

Beckhoff. Digital Compact Servo Drives. URL: https : //www.beckhoff.com/ax51xx/, 2018a. Visited on Jul. 16, 2018.

Beckhoff. Servomotor. URL: https : / www . beckhoff . com/am8532/, 2018b. Visited on Jul. 16, 2018.

Comau Robotics. $\mathrm{Nj}$ 110-3.0. URL: http: //Www.comau.com/EN/our-competences/ robotics/robot-team/nj-110-30, 2018. Visited on Jul. 16, 2018.

Denavit, J. and Hartenberg, R. S. A kinematic notation for lower-pair mechanisms based on matrices. Trans ASME J. Appl. Mech, 1955. 23:215-221.

Fossen, T. I. Handbook of Marine Craft Hydrodynamics and Motion Control. Wiley, 2011.

Gustafsson, T. Modelling and Control of Offshore Crane Systems. Ph.D. thesis, Luleå University of Technology, 1993.

Hartenberg, R. S. and Denavit, J. Kinematic synthesis of linkages. McGraw-Hill, 1964.

Heng, O. and Tørdal, S. S. Calibration of the norwegian motion laboratory using conformal geometric algebra. In CGI. 2017.

Hexagon Manufacturing Intelligence. Leica Absolute Tracker AT960. URL: http: //www . hexagonmi.com/ en-IN/products/laser-tracker-systems/, 2018. Visited on Jul. 16, 2018.

Kjelland, M. B. Offshore Wind Turbine Access Using Knuckle Boom Cranes. Ph.D. thesis, University of Agder, Grimstad, Norway, 2016.

Kongsberg Seatex. Kongsberg Seatex Motion Reference Unit. URL: https://www.km.kongsberg. com/, 2018. Visited on Jul. 16, 2018.

Luxcey, N., Reinholdtsen, S.-A., Sauder, T., Fouques, S., Jin, J., Kauczynski, W., and Hovland, G. Influence of wave-induced skid motions on the launch of free-fall skid lifeboats from floating hosts: Experimental and numerical investigations. 2014 . doi:10.1115/OMAE2014-24644.

Norwegian Motion Laboratory. URL: https://www . motion-lab.no/, 2018. Visited on Jul. 16, 2018.
Norwegian Research Council. Norwegian Roadmap for Research Infrastructure. URL: https://www.forskningsradet.no/ prognett-infrastruktur/Norwegian_Roadmap_ for_Research_Infrastructure/1253976312605, 2016. Visited on Aug. 17, 2018.

Qualisys. Qualisys. URL: https://www.qualisys. com/, 2018. Visited on Jul. 16, 2018.

Riverbank Computing Limited. Python PyQt5. URL: https://pypi.python.org/pypi/PyQt5/5. 9.1, 2018. Visited on Jul. 27, 2018.

Siciliano, B., Sciavicco, L., Villani, L., and Oriolo, G. Robotics: Modelling, Planning and Control. Advanced Textbooks in Control and Signal Processing. Springer London, 2010. doi:10.1007/978-1-84628642-1.

Spong, M., Hutchinson, S., and Vidyasagar, M. Robot Modeling and Control. Wiley, 2005. doi:10.1108/ir.2006.33.5.403.1.

Tørdal, S. S. and Hovland, G. Inverse kinematic control of an industrial robot used in vessel-to-vessel motion compensation. In 2017 25th Mediterranean Conference on Control and Automation (MED). pages 1392-1397, 2017a. doi:10.1109/MED.2017.7984313.

Tørdal, S. S. and Hovland, G. Relative Vessel Motion Tracking using Sensor Fusion, Aruco Markers, and MRU Sensors. Modeling, Identification and Control, 2017b. 38(2):79-93. doi:10.4173/mic.2017.2.3.

Tørdal, S. S., Hovland, G., and Tyapin, I. Efficient implementation of inverse kinematics on a 6dof industrial robot using conformal geometric algebra. Advances in Applied Clifford Algebras, 2017a. 27(3):2067-2082. doi:10.1007/s00006-016-0698-2.

Tørdal, S. S., Løvsland, P.-O., and Hovland, G. Testing of wireless sensor performance in vessel-to-vessel motion compensation. In Ind. Elec. Soc., IEEE IECON 2016-42nd Ann. Conf. pages 654-659, 2016. doi:10.1109/IECON.2016.7793951.

Tørdal, S. S., Pawlus, W., and Hovland, G. Realtime 6-dof vessel-to-vessel motion compensation using laser tracker. In OCEANS 2017 - Aberdeen. pages 1-9, 2017b. doi:10.1109/OCEANSE.2017.8084756. 


\section{A. Specification Tables}

Table 6: Comau SMART 5 - Technical Specification (Comau Robotics, 2018).

\begin{tabular}{ll}
\hline Description & Value \\
\hline Number of axes & 6 \\
Maximum wrist payload $[\mathrm{kg}]$ & 110 \\
Additional load on forearm $[\mathrm{kg}]$ & 50 \\
Maximum horizontal reach $[\mathrm{mm}]$ & 2980 \\
\hline Torque on axis $4[\mathrm{Nm}]$ & 638 \\
Torque on axis $5[\mathrm{Nm}]$ & 638 \\
Torque on axis $6[\mathrm{Nm}]$ & 314 \\
Stroke on Axis $1[\mathrm{deg}]$ & \pm 180 \\
Stroke on Axis $2[\mathrm{deg}]$ & $-75,+95$ \\
Stroke on Axis $3[\mathrm{deg}]$ & $-10,-256$ \\
Stroke on Axis $4[\mathrm{deg}]$ & \pm 280 \\
Stroke on Axis $5[\mathrm{deg}]$ & \pm 120 \\
Stroke on Axis $6[\mathrm{deg}]$ & \pm 2700 \\
Speed on Axis $1[\mathrm{deg} / \mathrm{s}]$ & 110 \\
Speed on Axis $2[\mathrm{deg} / \mathrm{s}]$ & 110 \\
Speed on Axis $3[\mathrm{deg} / \mathrm{s}]$ & 110 \\
Speed on Axis $4[\mathrm{deg} / \mathrm{s}]$ & 190 \\
Speed on Axis $5[\mathrm{deg} / \mathrm{s}]$ & 190 \\
Speed on Axis $6[\mathrm{deg} / \mathrm{s}]$ & 230 \\
Repeatability $[\mathrm{mm}]$ & 0.07 \\
Robot weight $[\mathrm{kg}]$ & 1070 \\
Tool coupling flange & ISO 9409 \\
\hline
\end{tabular}

Table 8: Specification and Capacity for E-Motion 8000 and E-Motion 1500 (Norwegian Motion Laboratory, 2018).

\begin{tabular}{lll}
\hline Description & EM 8000 & EM 1500 \\
\hline Max. Payload $[\mathrm{kg}]$ & $m=5500$ & $m=1500$ \\
Max. inertia $\left[\mathrm{kgm}^{2}\right]$ & $I_{x x}=21274$ & $I_{x x}=2023$ \\
Max. inertia $\left[\mathrm{kgm}^{2}\right]$ & $I_{y y}=24193$ & $I_{y y}=3713$ \\
Max. inertia $\left[\mathrm{kgm}^{2}\right]$ & $I_{z z}=28197$ & $I_{z z}=3611$ \\
\hline Surge $[\mathrm{m}]$ & $-1.110,+1.333$ & $-0.602,+0.716$ \\
Sway $[\mathrm{m}]$ & \pm 1.149 & \pm 0.603 \\
Heave $[\mathrm{m}]$ & $-0.955,+0.885$ & $-0.422,+0.407$ \\
Roll $[\mathrm{deg}]$ & \pm 26.10 & \pm 27.45 \\
Pitch $[\mathrm{deg}]$ & $-25.55,+33.40$ & $-24.35,+27.10$ \\
Yaw $[\mathrm{deg}]$ & \pm 31.10 & \pm 39.20 \\
\hline Surge $[\mathrm{m} / \mathrm{s}]$ & \pm 0.711 & \pm 0.8 \\
Sway $[\mathrm{m} / \mathrm{s}]$ & \pm 0.711 & \pm 0.8 \\
Heave $[\mathrm{m} / \mathrm{s}]$ & \pm 0.610 & \pm 0.6 \\
Roll $[\mathrm{deg} / \mathrm{s}]$ & \pm 20.0 & \pm 40.0 \\
Pitch $[\mathrm{deg} / \mathrm{s}]$ & \pm 20.0 & \pm 40.0 \\
Yaw $[\mathrm{deg} / \mathrm{s}]$ & \pm 20.0 & \pm 50.0 \\
\hline Surge $\left[\mathrm{m} / \mathrm{s}^{2}\right]$ & \pm 0.60 & \pm 0.65 \\
Sway $\left[\mathrm{m} / \mathrm{s}^{2}\right]$ & \pm 0.60 & \pm 0.60 \\
Heave $\left[\mathrm{m} / \mathrm{s}^{2}\right]$ & \pm 0.80 & \pm 0.60 \\
Roll $\left[\mathrm{deg} / \mathrm{s}^{2}\right]$ & \pm 100.0 & \pm 300.0 \\
Pitch $\left[\mathrm{deg} / \mathrm{s}^{2}\right]$ & \pm 100.0 & \pm 300.0 \\
Yaw $\left[\mathrm{deg} / \mathrm{s}^{2}\right]$ & \pm 100.0 & \pm 350.0 \\
\hline
\end{tabular}

Table 7: Comau SMART 5 - Geometric Dimensions (Notation According to Figure 12).

\begin{tabular}{lcc}
\hline Notation & Value & Unit \\
\hline$a_{1}$ & 0.350 & {$[\mathrm{~m}]$} \\
$d_{1}$ & 0.830 & {$[\mathrm{~m}]$} \\
$a_{2}$ & 1.160 & {$[\mathrm{~m}]$} \\
$a_{3}$ & 0.250 & {$[\mathrm{~m}]$} \\
$d_{4}$ & 1.492 & {$[\mathrm{~m}]$} \\
$d_{6}$ & 0.210 & {$[\mathrm{~m}]$} \\
$d_{t p}$ & 0.567 & {$[\mathrm{~m}]$} \\
\hline
\end{tabular}

Table 9: Technical Information of the Beckhoff Servomotor (AM8532-H). Data for $400 \mathrm{~V} \mathrm{AC}$ (Beckhoff, 2018b).

\begin{tabular}{lcl}
\hline Description & Value & Unit \\
\hline Standstill Torque & 2.37 & {$[\mathrm{Nm}]$} \\
Rated Torque & 1.85 & {$[\mathrm{Nm}]$} \\
Rated Speed & 9000 & {$[\mathrm{rpm}]$} \\
Rated Power & 1.74 & {$[\mathrm{~kW}]$} \\
Peak Torque & 11.65 & {$[\mathrm{Nm}]$} \\
Standstill Current & 5.10 & {$[\mathrm{~A}]$} \\
Peak Current & 29.5 & {$[\mathrm{~A}]$} \\
Torque Constant & 0.46 & {$[\mathrm{Nm} / \mathrm{A}]$} \\
Voltage Constant & 32 & {$[\mathrm{mV} / \mathrm{min}]$} \\
Number of poles & 8 & {$[-]$} \\
Rotor moment of inertia & 2.05 & {$\left[\mathrm{kgcm}^{2}\right]$} \\
Brake moment of inertia & 0.10 & {$\left[\mathrm{kgcm}^{2}\right]$} \\
\hline
\end{tabular}

